\title{
Influência dos Procedimentos e Modos de Transferência no processo MAG na Redução de Deformações em Painéis de Estruturas Navais
}

\author{
(Influence of Procedure and Different Transfer Modes in MAG in Welding Process in Deformations Reduction of Marine \\ Structures Panels)
}

\author{
Tárcio dos Santos Cabral ${ }^{1}$, Eduardo de Magalhães Braga², Everton Augusto Maciel Mendonça ${ }^{3}$, Américo Scotti ${ }^{4}$, \\ 1,2,3 Universidade Federal do Pará, Departamento de Engenharia Mecânica, Belém, Pará,_Brasil, tarcio_cabral@yahoo.com.br, \\ edbraga@ufpa.br,notreve@ufpa.br \\ ${ }^{4}$ Universidade Federal de Uberlândia, Departamento de Engenharia Mecânica, Uberlândia, Minas Gerais, Brasil, ascotti@ufu.br
}

\begin{abstract}
Resumo
Juntamente com o crescimento da indústria naval brasileira surgiram problemas provenientes dos processos de produção na forma de distorções e tensões residuais. Neste trabalho foi estudada a influência de procedimentos de soldagem (sequência e direção de soldagem e uso do passe reverso) e modos de transferência (MAG-CC, MAG-STT e MAG-CW) sobre os valores de distorções. Todas as soldagens foram realizadas com o arame ER70S-6 e gás de proteção $\mathrm{CO}_{2}$ puro no modo curto-circuito. Ao final das soldagens, observou-se que a variável mais importante foi a mudança de direção de soldagem, que está ligada diretamente aos níveis de restrição do painel teste. As sequências utilizadas surtiram pouco efeito nos valores de distorção. O passe reverso obteve valores baixos de distorção, contudo, mostrou-se improdutivo. Observou-se que os valores de distorção para os processos e MAG-STT resultaram em valores de distorção semelhantes. Os painéis soldados pelo processo MAG-CW obtiveram os menores valores de distorção mensurados.
\end{abstract}

Palavras-chave: Distorções. MAG. MAG-STT. MAG-CC. Cold Wire.

\begin{abstract}
The Growth in the Brazilian shipbuilding industry brought on numerous problems in the production line. Some of these problems are related to the manufacturing process, welding process, due to residual stress and distortions. In the present paper, the influence of the welding procedure (sequence and direction of welding and the use of back weld) was studied. Different welding processes with different transfer modes (GMAW-CC, GMAW-TT and GMAW-CW) were also used. These changes were made to observe the influences on the amplitude of the distortions. All the welds were made using the ER70S-6 electrode and pure CO, as a shielding gas with the short circuit transfer mode. The results showed that the most important variable is the direction of welding, which is related to the level of restriction of the panels welded. The effect of the welding sequence presented on the values of distortion produced were minor. The panels produced using the back welding procedure presented small values of distortion however, the procedure showed to be inefficient. It was also observed that the level of distortion for the processes GMAW-CC and GMAW-STT showed similar values. On the other hand the values of distortion in the panel welded using the GMAW-CW process presented the lowest values.
\end{abstract}

Key-words: Distortion. GMAW. GMAW-STT. GMAW-CC. Cold Wire.

\section{Introdução}

O processo de soldagem é o principal responsável pelo surgimento das imperfeições de fabricação [1], contudo, não se pode desconsiderar que as formas de transporte e armazenamento das chapas contribuem com uma parcela na distorção final dos painéis enrijecidos de estruturas navais. As estruturas navais são constituídas, basicamente, de painéis enrijecidos, que formarão blocos e estes darão forma às construções. Os painéis são formados pela união de chapas que são enrijecidas pelo processo

Recebido em 22/07/2013, texto final em 30/09/2013. de soldagem de reforços longitudinais e transversais. Para que haja o processo de união, deve ser aplicado calor, porém, uma parcela desse calor não homogêneo, aplicado ao material durante a soldagem, gera uma expansão térmica ao redor da área do cordão de solda. Esse efeito pode ser visualizado na Figura 1. A barra central aquecida na Figura 1A representa o cordão de solda depositado, já na Figura 1B é feito um comparativo entre o mecanismo de barras e uma placa soldada. Como o cordão, inicialmente, é a única parcela da placa aquecida, irá se expandir com um determinado nível de energia e as regiões ao redor tenderão a se manter imóveis, bloqueando a dilatação. Em um segundo momento, na fase de resfriamento, a placa irá se contrair e, novamente, as regiões ao redor irão restringir essa contração. Esse processo de dilatação e contração gera as distorções e tensões residuais provenientes do processo de soldagem. 
As imperfeições devido à deformação podem ocasionar graves problemas estruturais, entre os quais se destacam a formação de trincas, maior tendência à fratura frágil e falta de estabilidade dimensional. Autores que analisaram em seus estudos o fenômeno das distorções concordam que além de se reduzir a resistência mecânica das estruturas navais, a distorção afeta o processo de fabricação, aumentando o tempo e o custo de produção devido, principalmente, às necessidades de retrabalho nas estruturas [1]; [2] e [3].
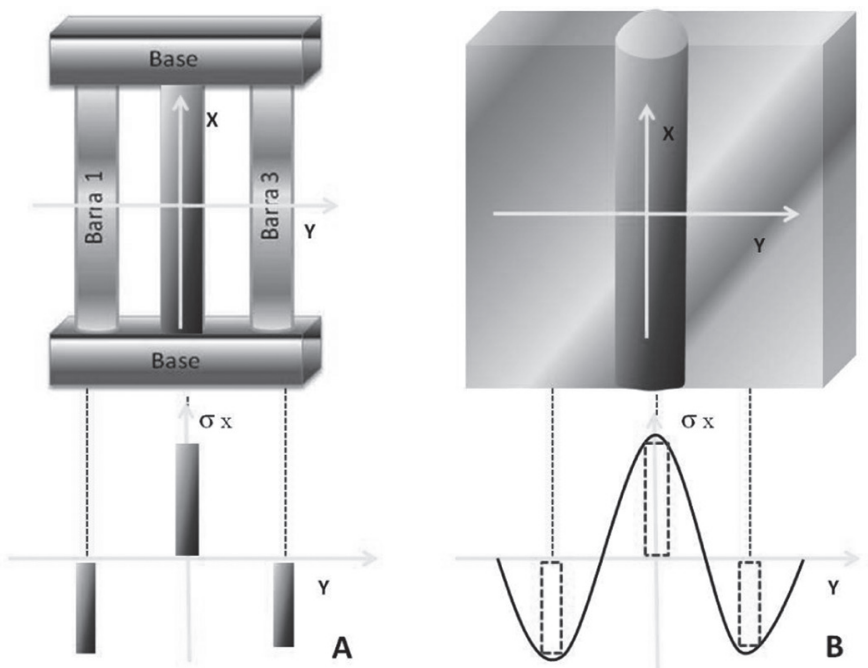

Figura 1. Comparação entre as tensões residuais e a montagem de três barras (A) e as tensões residuais longitudinais formadas ao longo da direção transversal y de uma solda de topo (B)

A construção das estruturas navais é caracterizada pela produção em série, e é nesse estágio que se deve aplicar procedimentos que irão promover um menor nível de distorção nos painéis. Tais procedimentos podem ser a mudança do modo de transferência da soldagem, emprego de uma sequência de soldagem mais eficiente, automação da linha de montagem dos painéis, dentre outros.

Os modos de transferência que foram utilizados neste estudo são o MAG em curto-circuito (MAG-CC), que corresponde ao um dos modos de transferência mais usuais na construção de estruturas metálicas. Segundo [4]; [5] e [6] esse modo trabalha em baixos valores de corrente e tensão, e produz uma poça de fusão relativamente pequena, de rápida solidificação, além disso, como as gotas são transferidas pelo contato com a poça de fusão, através da tensão superficial, é possível a soldagem em todas as posições.

Outro modo de transferência que foi utilizado foi o modo de curto-circuito controlado, conhecido como Surface Tension Transfer (MAG - STT). Esse modo de transferência utiliza uma fonte de soldagem com inversores de alta frequência juntamente com controle avançado da forma de onda para produzir soldagens com alta qualidade, diminuindo significativamente a geração de fumos e de respingos. O modo STT inicialmente foi empregado na indústria automobilística, na soldagem de chapas de pequena espessura, já que o controle da corrente permite monitorar o aporte de calor na poça de fusão. Posteriormente, após pesquisas, começou a ser empregado na soldagem de passes de raiz em dutos [7]. A forma de onda gerada pelo processo STT, de modo geral, pode ser visualizada na Figura 2.

Já no modelo de soldagem MAG com adição de arame não energizado ou "arame frio" (MAG-CW), o procedimento consiste na introdução de um arame "frio" na atmosfera do arco voltaico gerado pelo arame energizado. Deste modo, o arame frio funde-se - aproveitando o calor do arco elétrico juntamente com o arame energizado gerando o metal de adição [3]. O modelo de transferência MAG-CW está visualizado esquematicamente na Figura 3.

Desta forma, o presente estudo se propõe a analisar as distorções provenientes da mudança do procedimento de soldagem através da construção de um modelo de painel enrijecido, empregando sequências de soldagens previamente estabelecidas, mudança da direção de soldagem e dos modos de transferência MAG curto-circuito - (MAG-CC), MAG Surface Tension Transfer (MAG-STT) e o MAG-CW.

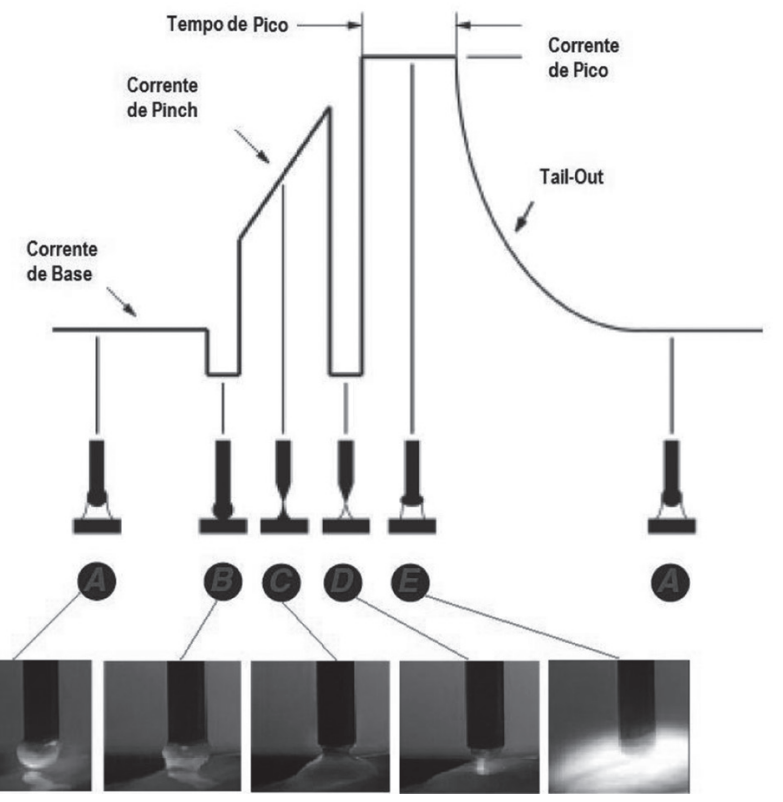

Figura 2 - Esquema Gráfico da Forma de Onda do modo de transferência STT, Modificado de [15]

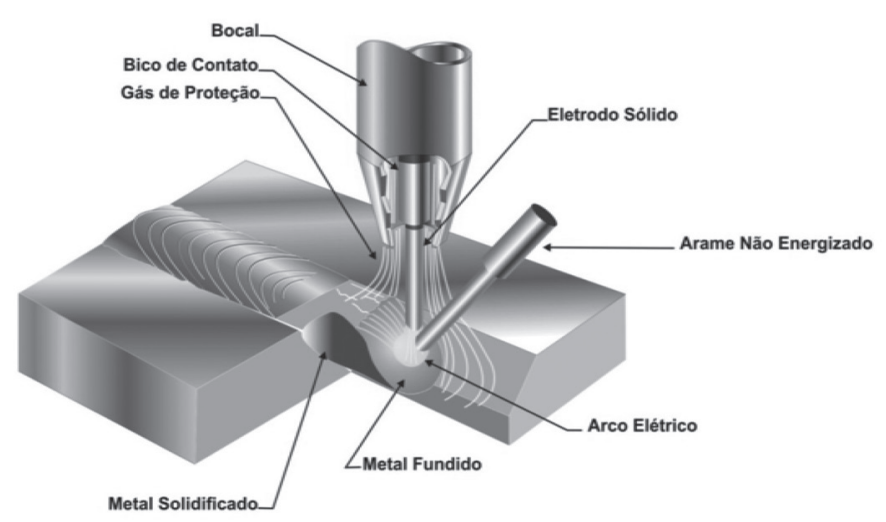

Figura 3 - Desenho esquemático do processo MIG/MAG com adição de arame frio, [17] 


\section{Materiais e Métodos}

Os parâmetros utilizados para a comparação dos resultados finais de distorção foram: o valor de corrente, que está diretamente ligado aos níveis de aporte líquido de calor; a relação de deposição utilizada na soldagem, para se obter um mesmo volume depositado em cada cordão e manter os mesmos níveis de distorção; mesmos consumíveis utilizados (material de adição, gás de proteção, chapas e cantoneiras que formaram o painel enrijecido); mesmo comprimento de cordão e mesmo ângulo de trabalho.

\subsection{Metodologia}

\subsubsection{Determinação dos Parâmetros Para o Processo MAG- $\mathrm{CC}$}

Depois de encontrado o valor inicial de velocidade alimentação $3,9 \mathrm{~m} / \mathrm{min}$, que resultou em uma corrente de 170 A a uma velocidade de soldagem de $21 \mathrm{~cm} / \mathrm{min}$, estes valores proporcionaram um cordão com bom aspecto superficial. Foi proposta a utilização de uma Relação de Deposição (velocidade de alimentação do arame dividido pela velocidade de soldagem) conforme relação utilizada por [8]; [9]; [10]; [11] e [12]. Os valores encontrados nos estudos preliminares resultaram em uma constante no valor adimensional de $(18,6)$ que será utilizado para se encontrar a velocidade de alimentação e/ou soldagem para cada processo analisado.

As soldagens foram realizadas pelo processo $\mathrm{MAG}-\mathrm{CC}$, com gás de proteção $\mathrm{CO}_{2}$ puro, distância bico de contato peça (DBCP) de $12 \mathrm{~mm}$ e arame de soldagem ER70S-6 de 1,2 mm de diâmetro nominal no modo $\mathrm{CC}+$ com um ângulo de trabalho, tocha e a mesa de soldagem, de $23^{\circ}$. Para a determinação dos parâmetros de trabalho foram realizadas soldagens de forma contínua sobre chapas, variando-se o valor de velocidade de alimentação e tensão com uma condição cujo valor de tensão proporcionasse uma boa transferência metálica por curto-circuito. Essa análise foi realizada de uma forma simplificada, em que os recursos da visão e audição foram utilizados para se obter uma faixa de "estabilidade" para o processo. A faixa de tensão na qual se foi possível soldar ficou entre 21 a $26 \mathrm{~V}$.

Depois de encontrada a faixa de tensão, foi utilizada uma peça teste, utilizando o mesmo tipo de junta proposta para os painéis. Como não foi encontrado apenas um valor de tensão dito ótimo para a soldagem, fez-se necessário realizar o refino desses valores, para isso, recorrendo-se ao critério de estabilidade de transferência por curto-circuito, através de um índice de regularidade na transferência metálica (IVcc) [3]; [13] e [14] para se obter apenas um valor de tensão juntamente com a característica superficial e geométrica do cordão de solda. Pode ser observado na Tabela 1 o valor final dos parâmetros de soldagem para o processo MAG-CC.

\subsubsection{Determinação dos Parâmetros de Soldagem Para o Processo MAG-STT}

As variáveis operacionais do processo STT são a velocidade de alimentação de arame, corrente de pico, corrente de base e "Tail-out". A velocidade de alimentação controla a taxa de deposição. De acordo com a literatura [15], a corrente de pico controla o comprimento do arco e afeta a forma da face de raiz de solda. Já a corrente de base controla o calor imposto "fino", afetando a parte de trás do cordão, e por fim, o "Tail-out" controla o calor imposto "grosso". Testes foram realizados objetivando encontrar o valor de corrente média estabelecido anteriormente 170 A. Assim, o valor de velocidade de alimentação obtido foi de $4,1 \mathrm{~m} / \mathrm{min}$ e ao se utilizar a $\boldsymbol{R e l a c ̧ a ̃ o ~ d e ~ D e p o s i c ̧ a ̃ o ~ c i t a d a ~}$ anteriormente, foi encontrada a velocidade de soldagem, $22 \mathrm{~cm} /$ min. Para a manutenção desses valores adotados foi encontrada também a corrente de pico $300 \mathrm{~A}$, corrente de base 100 A e Tailout no valor de 5 .

Com base nesses valores, soldagens foram realizadas utilizando peças para teste semelhantes aos utilizados para se obter os parâmetros finais de soldagem do processo MAG-CC. O melhor resultado obtido pode ser visualizado na Tabela 2. A escolha destes parâmetros é derivada da característica superficial e geométrica do cordão de solda.

\subsubsection{Determinação dos parâmetros de soldagem para 0 processo MAG-CW}

Para a soldagem MAG-CC com injeção de arame frio (MAG$\mathrm{CW}$ ) foi mantido o valor de corrente utilizado nos procedimentos anteriores 170 A. Para isso foram realizados testes preliminares,

Tabela 1. Parâmetros de soldagem para o processo MAG-CC.

\begin{tabular}{ccccc}
\hline Valim. $(\mathrm{m} / \mathrm{min})$ & Vsold. $(\mathrm{cm} / \mathrm{min})$ & Tensão $(\mathrm{V})$ & DBCP $(\mathrm{mm})$ & V. G $(1 / \mathrm{min})$ \\
\hline 3,90 & 21,00 & 24,50 & 12,00 & 15 \\
\hline
\end{tabular}

Onde: Valim - velocidade de alimentação; Vsold - velocidade de soldagem, DBCP - distância bico de contato peça, V.G - Vazão de gás.

Tabela 2. Valores médios adquiridos nos testes preliminares MAG-STT.

\begin{tabular}{|c|c|c|c|c|c|c|c|c|}
\hline \multirow{2}{*}{ Ip (A) } & \multirow{2}{*}{$\mathrm{Ib}(\mathrm{A})$} & \multirow{2}{*}{ To } & \multicolumn{6}{|c|}{ Parâmetros } \\
\hline & & & Valim $(\mathrm{m} / \mathrm{min})$ & Vsold (cm/min) & $\operatorname{Im}(\mathrm{A})$ & $\mathrm{Um}(\mathrm{V})$ & IVcc & Freq $(\mathrm{Hz})$ \\
\hline 300 & 100 & 5 & 4,10 & 22,00 & 168,97 & 17,69 & 0,65 & 89,78 \\
\hline
\end{tabular}

Onde: To - Tail-out; Ip - corrente de pico; Ib - corrente de base; Im - corrente média; Um - tensão média; Freq - frequência de curtocircuito. 
semelhantes aos utilizados para os processos anteriores, em que foi encontrada a velocidade de alimentação de arame energizado de $3,73 \mathrm{~m} / \mathrm{min}$. Essa diminuição no valor de alimentação se deve ao fato que ao se manter a mesma velocidade de alimentação do processo MAG-CC ocorre um aumento do valor de corrente, saindo da faixa de trabalho estipulada para este trabalho. Dessa forma, o valor de alimentação foi reduzido até chegar ao valor de 170 A e o valor de alimentação escolhido para o arame não energizado foi de $2,00 \mathrm{~m} / \mathrm{min}$. Esse valor de velocidade de soldagem foi baseado em estudos anteriores, nos quais seu valor apresentou bons resultados de rendimento e geometria decorrentes dos trabalhos de [16] e [17].

De posse do valor de velocidade de alimentação e já sabendo a relação de deposição $(18,6)$, foi encontrada a velocidade de soldagem. Para encontrar essa velocidade foi proposto fazer a somatória das velocidades de alimentação (velocidade de alimentação do arame energizado com a velocidade de alimentação do arame não energizado), cujo resultado foi de 31 $\mathrm{cm} / \mathrm{min}$. O arame utilizado foi o ER70S-6, de diâmetro nominal de 1,2 mm como arame energizado no modo $\mathrm{CC}+$, e o arame de 1,0 mm diâmetro como arame frio. $\mathrm{O}$ ângulo de entrada do arame não energizado correspondeu a $43^{\circ} \mathrm{em}$ relação à tocha.

Assim como para o processo MAG-CC e MAG-CW, o critério de escolha dos parâmetros de soldagem inicia-se pela escolha da melhor geometria do cordão de solda e melhor índice de regularidade. Foram realizadas soldagens com uma variação de tensão de 22 a $26 \mathrm{~V}$, em que os melhores resultados de geometria foram colocados para análise. A análise dos valores obtidos através do programa para o cálculo do IVcc ocorreu igualmente ao do processo MAG-CC. A Tabela 3 apresenta os parâmetros de soldagem adotados para o processo MAG-CW.

Tabela 3. Parâmetros de soldagem para o processo MAG-CC.

\begin{tabular}{ccccc}
\hline $\begin{array}{c}\text { Valim. } \\
(\mathrm{m} / \mathrm{min})\end{array}$ & $\begin{array}{c}\text { Vsold. } \\
(\mathrm{cm} / \mathrm{min})\end{array}$ & $\begin{array}{c}\text { Tensão } \\
(\mathrm{V})\end{array}$ & $\begin{array}{c}\text { DBCP } \\
(\mathrm{mm})\end{array}$ & $\begin{array}{c}\text { V. G (l/ } \\
\mathrm{min})\end{array}$ \\
\hline 3,73 & $\mathbf{3 1 , 0 0}$ & $\mathbf{2 5 , 0 0}$ & $\mathbf{1 2 , 0 0}$ & 15 \\
\hline
\end{tabular}

Onde: Valim - velocidade de alimentação; Vsold velocidade de soldagem, DBCP - distância bico de contato peça, V.G - Vazão de gás.

\subsubsection{Planejamento Experimental para Análise da Distorção dos Painéis}

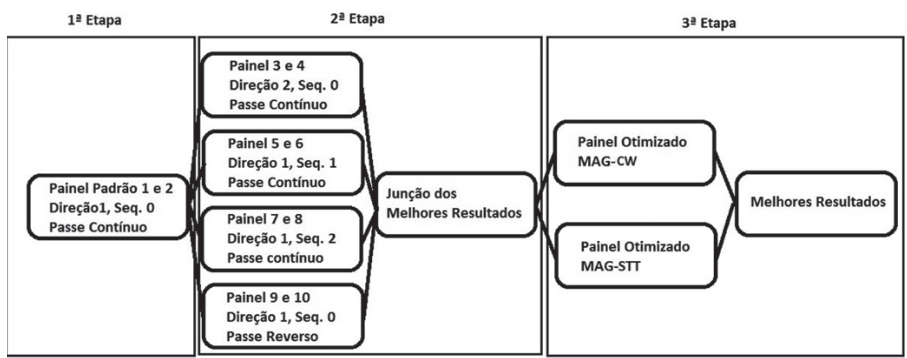

Figura 4. Fluxograma das etapas a serem realizadas neste trabalho
A fim de se maximizar as análises deste trabalho foi desenvolvido um fluxograma, descrito na Figura 4, no qual se pode observar as etapas distintas que foram realizadas. Neste sentido, o planejamento busca a otimização dos procedimentos e modos de transferência da soldagem MAG. Cada etapa é descrita com mais detalhes logo em seguida.

i. Primeira Etapa: Busca das dimensões para os painéis enrijecidos que garanta uma boa visualização das distorções resultantes do processo de soldagem. Escolha de três sequências de soldagem, sendo que uma delas será mantida como sequência padrão para as demais soldagens e as outras duas serão variações do planejamento experimental. Por fim será formulado um pacote operacional para as soldagens em curto-circuito.

ii.Segunda Etapa: Realização de soldagens pelo processo MAG - CC com as variações operacionais estipuladas para o estudo das distorções nos painéis:

- Sentido de soldagem;

- Sequências de soldagem diferentes das adotadas no painel padrão; e

- Soldagem com passe reverso.

iii. Terceira Etapa: Nesta etapa após a soldagem das variações, foram unificados os melhores resultados na soldagem de um painel otimizado. Foram formulados pacotes operacionais para os processos MAG-CW e MAG- STT. As soldagens realizadas para cada parâmetro geraram dois corpos de prova para cada parâmetro que foi estudado neste trabalho.

\subsubsection{Sequências de Soldagem Para os Painéis Enrijecidos}

Tomando como base para aplicação das sequências de soldagem foram adotados critérios de [18], que para uma dada estrutura soldada constituída de várias uniões, a sequência de soldagem deve estar intimamente ligada à sequência de montagem. Para isso devem ser seguidas algumas regras básicas: manter as extremidades da soldagem livre de fixação, começar o processo de soldagem pelas juntas que irão contrair mais e as soldas devem ser aplicadas em simetria com seu eixo neutro sempre que possível.

Neste trabalho foi adotada uma sequência como padrão, e as outras duas sequências foram derivadas da primeira. A sequência inicial, sequência zero, foi idealizada para distribuir as soldagens pelos quadrantes do painel, distribuir o calor gerado uniformemente sobre a peça e assim distribuir as distorções geradas. A sequência um foi utilizada para depositar os cordões ao redor do painel, como uma "espiral", de forma que a cada dois passes, seria fixado um enrijecedor. A sequência dois foi utilizada para proporcionar que cada quadrante pudesse expandir com maior mobilidade. Podem ser vistas nas Figuras 5 a 9 as sequências e as direções de soldagem dos painéis teste.

Em cada painel serão depositados seis cordões de solda para fixação dos enrijecedores, respeitando a ordem e a sequência de soldagem. Cada cordão de solda terá cerca de $14 \mathrm{~cm}$ de comprimento e ficará distanciado do centro do painel cerca 


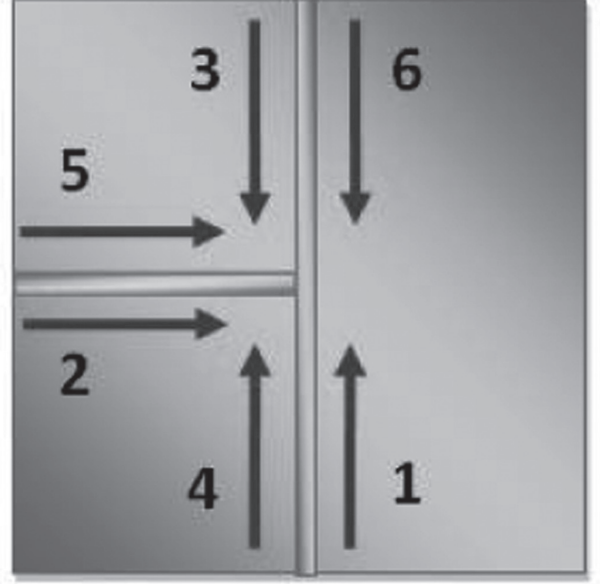

Figura 5. Sequência Zero/ Para Dentro do Painel/ Passe Contínuo

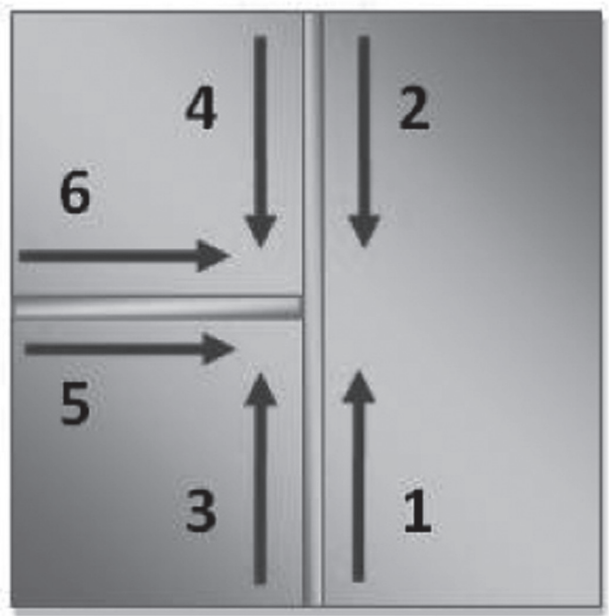

Figura 8. Sequência Dois/ Para Dentro do Painel/ Passe Contínuo

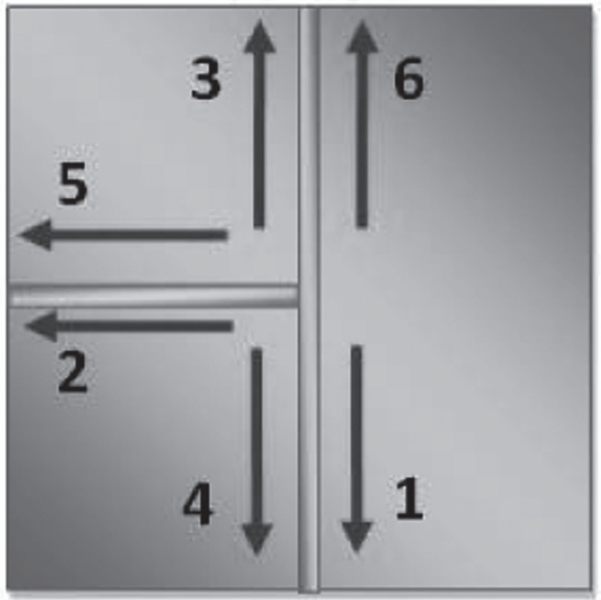

Figura 6. Sequência Zero/ Para Fora do Painel/ Passe Contínuo

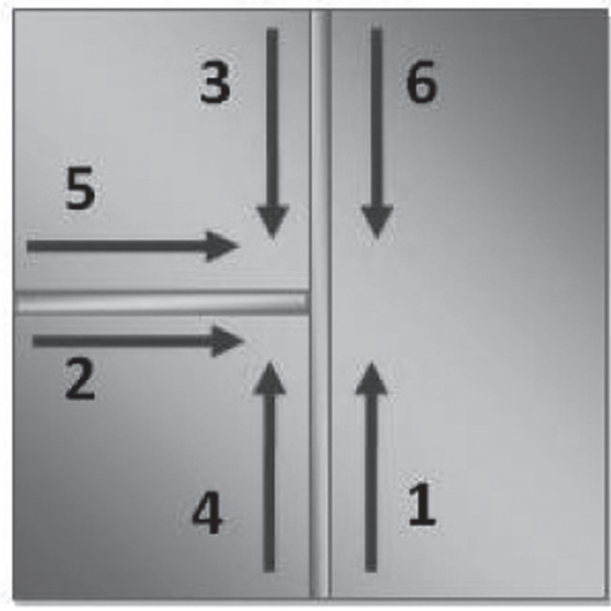

Figura 9. Sequência Zero/ Para Dentro do Painel/ Passe Reverso

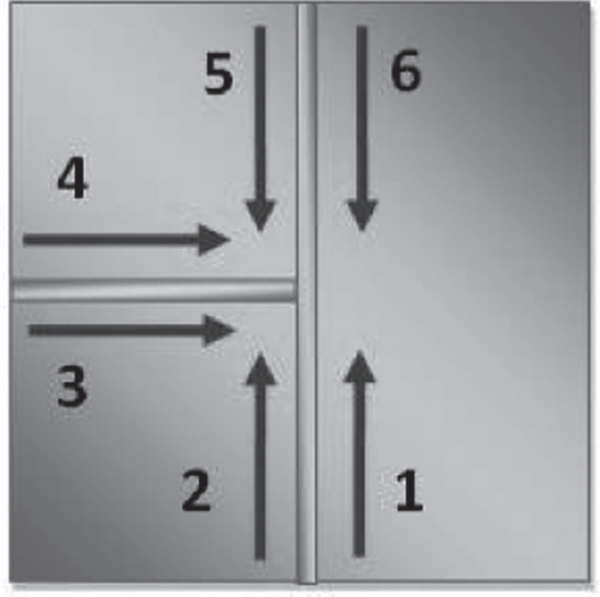

Figura 7. Sequência Um/ Para Dentro do Painel/ Passe Contínuo

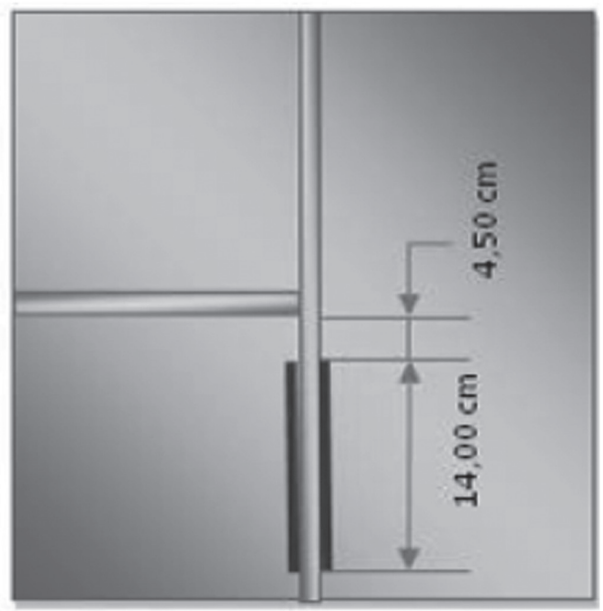

Figura 10. Esquema do comprimento do cordão de solda de 4,5 cm, conforme apresentado na Figura 10. Cada placa de aço baixo carbono ABNT 1020 utilizada nos painéis terá as dimensões de $400 \times 400 \times 3,2$ mm (largura x comprimento $x$ espessura) e o enrijecedor utilizado foram 2 peças de uma cantoneira em "L" simétrico de 1 " x 3/16", uma com 400 $\mathrm{mm}$ de comprimento e a outra com $195 \mathrm{~mm}$ de comprimento. A disposição dos enrijecedores pode ser visualizada na Figura 8b. Para a soldagem com a utilização do passe reverso, o cordão foi dividido em três segmentos de 4,66 cm de comprimento. Os passes de solda depositada seguem a ordem numérica crescente vista no esquema das Figuras 5 a 10, cuja ordem foi utilizada para os demais painéis. As setas em destaque representam a direção de soldagem.

\subsubsection{Tomada dos Valores de Distorção}

Para a tomada dos valores de distorção dos painéis se faz necessário estipular um padrão de medida. Para isso, foram feitas

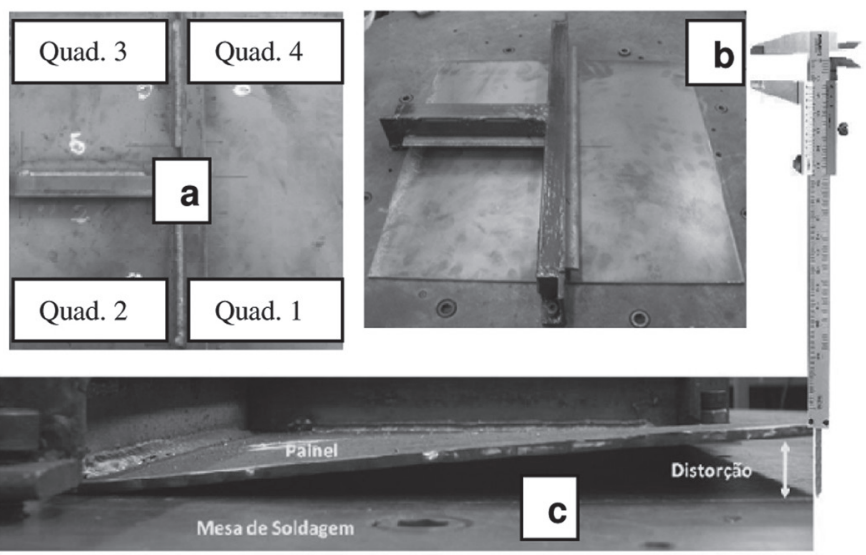

Figura 11. Visão geral do painel enrijecido. (a) Painel livre após a soldagem com seus quadrantes, (b) Painel fixo na mesa se soldagem através do suporte e (c) esquema da localização

do local onde foram retiradas as medidas de distorção 
várias considerações: o corpo de prova foi dividido em quatro quadrantes (Figura 11), e em cada canto do painel foi realizada a tomada dos valores de distorção. A mesa de soldagem serviu de referência para a retirada das medidas, devido ao fato de ser robusta e manter boa planicidade. As dimensões das distorções foram obtidas através de um paquímetro com resolução de $1 / 20$ na temperatura ambiente e com a peça fixa no suporte, conforme indicado na Figura 11.

\subsection{Materiais}

\subsubsection{Célula de Trabalho}

A célula de trabalho foi elaborada com o objetivo de se realizar as sequências, mudança de direção e mudança de modo de deposição de soldagem, para manter o mesmo padrão de comportamento requerido na execução dos experimentos. Devido a esta necessidade foi empregado um braço robótico para se efetuar os movimentos da tocha.

\subsubsection{Painéis Enrijecidos}

Para o dimensionamento dos painéis enrijecidos foi necessário realizar soldagens exploratórias e a escolha do modelo final baseou-se nos seguintes critérios: proporcionalidade das dimensões (real x modelo); dimensões e fixação na mesa de soldagem; sensibilidade do painel às deformações geradas; repetibilidade nos valores de distorção; e o transporte e armazenamento.

A avaliação do efeito dos processos de soldagem e procedimentos (sequência de passes e mudança de direção) sobre a deformação foi realizada inicialmente em uma peça de teste, para simular a situação real de um painel naval, que fosse suficientemente pequena (viável economicamente), e ao mesmo tempo realista, que tivesse sensibilidade para refletir os efeitos da distorção. Depois de várias propostas, optou-se pela peça

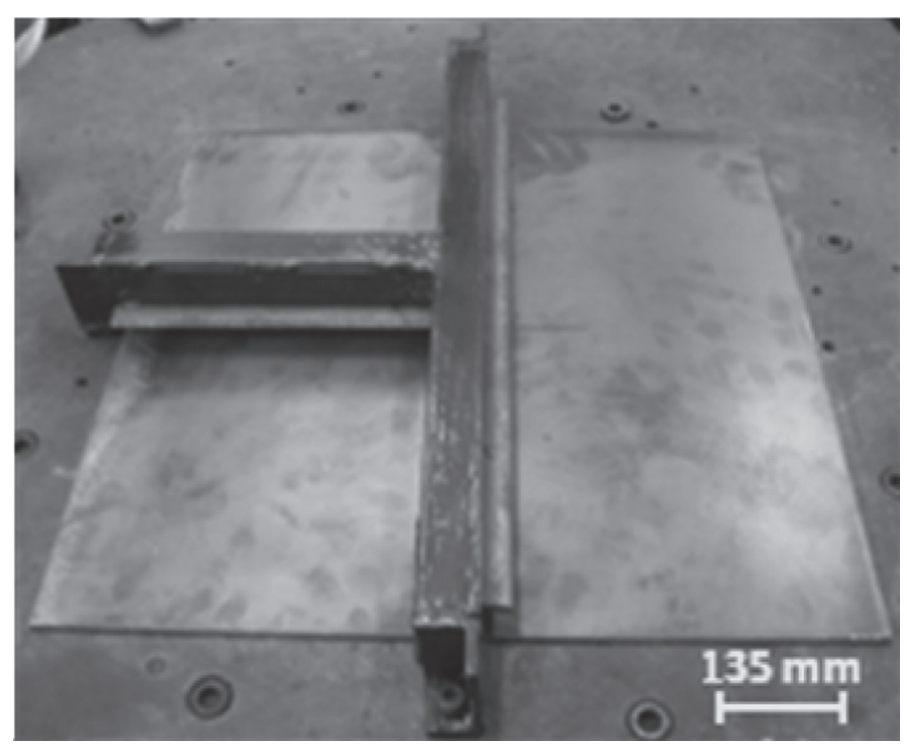

Figura 12. Imagem do modelo de painel enrijecido confeccionado para os testes

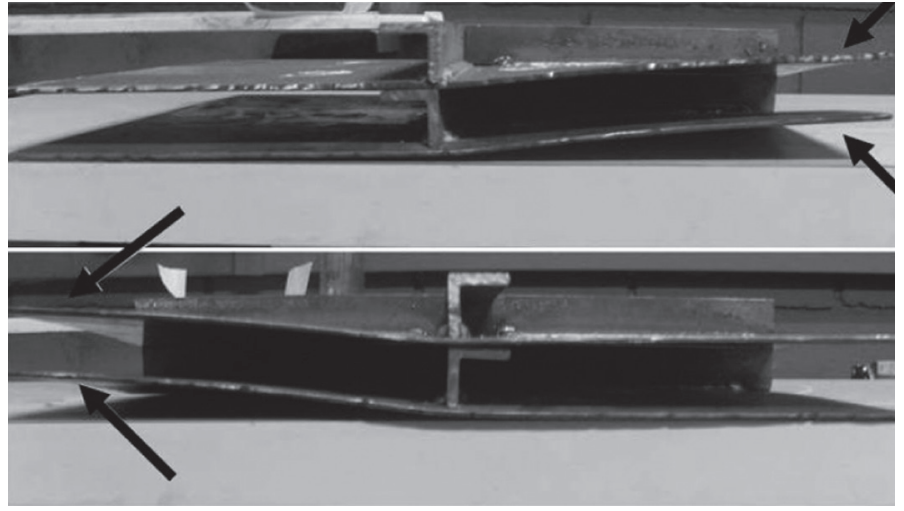

Figura 13. Exemplo de distorção nos painéis enrijecidos com vários ângulos de visão

de teste vista na Figura 12. Uma série de testes foi realizada para validação do painel, conforme observado na Figura 13, na qual se pode verificar que o painel possui a sensibilidade e repetibilidade necessárias para se efetuar os ensaios.

Segundo [18] e [19] e, o ponteamento inicial gera tensões residuais, provenientes do travamento das chapas, impedindo o deslocamento de expansão. Desta forma, foi proposta a não utilização do ponteamento nos enrijecedores nas chapas, que foram fixadas através de um suporte sobre pressão, visto na Figura 12, a fim de se manter o deslocamento de expansão das chapas durante o processo de aquecimento.

\section{Resultados e Discussão}

\subsection{Resultados da Primeira Etapa}

Um dos objetivos dessa etapa foi encontrar um modelo de painel enrijecido para a realização das soldagens pelo processo MAG-CC, conforme foi visto na Figura 13. As soldagens do painel padrão no modo MAG-CC foram realizadas baseadas no pacote operacional, descrito no item 2.1.1.

\subsection{Resultados da Segunda Etapa}

O objetivo da segunda etapa foi a realização das soldagens MAG-CC com as variações operacionais estipuladas para o estudo das distorções. Após todas as variáveis encontradas, pode-se dar início à soldagem dos painéis teste. Cada variável gerou dois painéis, formando um total de dez painéis ao final da etapa. Em geral, o processo teve incidência de salpicos normais para o processo curto-circuito com proteção gasosa de $\mathrm{CO}_{2}$ puro.

Após a realização das soldagens da segunda etapa, os resultados das distorções foram relacionados tomando como referência a distorção global de todos os quadrantes dos painéis soldados, ou seja, foram somados todos os valores de distorção obtidos. Desses resultados foi retirada uma sequência e uma direção "ótima" e um tipo de passe para a soldagem do painel otimizado. Pode-se observar na Tabela 4 as variações utilizadas na segunda etapa. 
Tabela 4. Variáveis de análise da segunda etapa

\begin{tabular}{|c|c|c|}
\hline Exp. & Variáveis & Processo \\
\hline $\mathbf{1}$ & $\begin{array}{c}\text { Sequência Zero/ Para Dentro do Painel/ } \\
\text { Passe Contínuo }\end{array}$ & MAG-CC \\
\hline $\mathbf{2}$ & $\begin{array}{c}\text { Sequência Zero/ Para Fora do Painel/ } \\
\text { Passe Contínuo }\end{array}$ & MAG-CC \\
\hline $\mathbf{3}$ & $\begin{array}{c}\text { Sequência Um/ Para Dentro do Painel// } \\
\text { Passe Contínuo }\end{array}$ & MAG-CC \\
\hline $\mathbf{4}$ & $\begin{array}{c}\text { Sequência Dois/ Para Dentro do Painel// } \\
\text { Passe Contínuo }\end{array}$ & MAG-CC \\
\hline $\mathbf{5}$ & $\begin{array}{c}\text { Sequência Zero/ Para Dentro do Painel// } \\
\text { Passe Reverso }\end{array}$ & MAG-CC \\
\hline
\end{tabular}

As medidas foram retiradas das pontas dos painéis conforme item 2.1.6. Pode ser visualizado na Figura 14 o valor da distorção global dos cinco primeiros experimentos com o respectivo valor de desvio padrão.

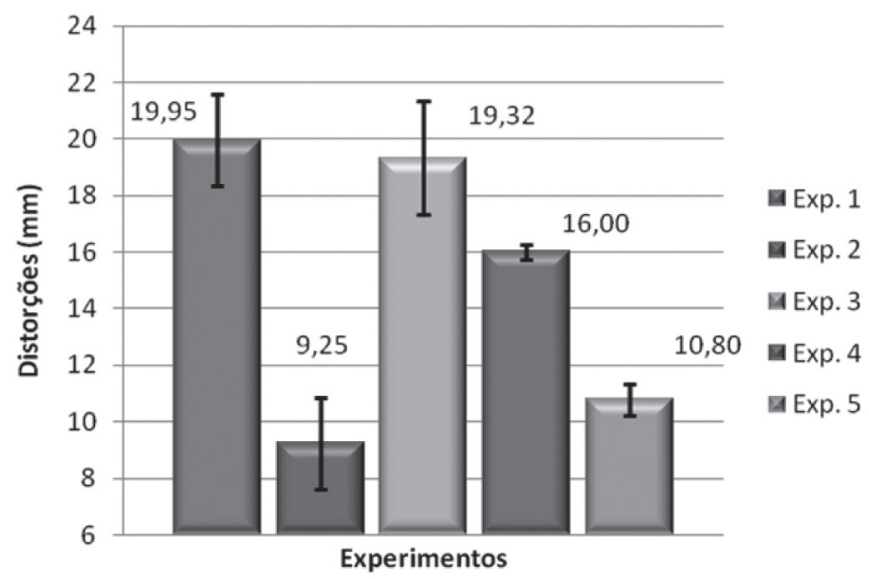

Figura 14. Distorção global dos experimentos da segunda etapa

Foi possível observar pelo gráfico da Figura 14 que o menor valor de distorção obtido pelos procedimentos adotados foi o correspondente ao experimento 2 , que foi $53,64 \%$ menor que o experimento que obteve o maior valor de distorção (experimento 1). Outro bom resultado correspondeu ao experimento 5 (passe reverso), que em relação ao experimento 1 foi $46,36 \%$ menor. $\mathrm{O}$ experimento 3 obteve um valor muito próximo ao obtido no experimento 1 , já o experimento 4 , apesar de apresentar um valor menor que o relativo ao experimento 1 e 3 , ainda foi superior ao experimento 2 e 5 .

\subsubsection{Distorção Relacionada com Mudança de Direção de Soldagem e Passe Reverso}

Foram medidos os valores de distorção (Figura 14) em que se pode notar que os menores valores de distorção são provenientes do passe dado na direção centro/extremidade (painel 2) e o passe reverso (painel 5). Esse resultado assemelha-se aos resultados obtidos nos estudos numéricos de [20], que obteve melhores valores de distorção utilizando dois passes geometricamente opostos partindo do centro de uma junta em "T", do que em um único passe, que partiu de uma das bordas da sua peça de trabalho.

Em termos de produtividade, a mudança de direção de soldagem não acarreta mudanças sérias na montagem dos aparatos de soldagem, contudo, a utilização de outra técnica de soldagem, o passe reverso, (painel 5), irá fornecer valores baixos de distorção devido a um maior controle do diferencial de temperatura aplicado sobre a chapa soldada e uma distribuição mais uniforme de tensões residuais [18] e [20]. Todavia, esse ganho nos valores de distorção deverá ser bem ponderado, visto que para obtê-los foi empregado um maior tempo na execução das soldagens.

A segmentação do cordão em partes menores acarreta uma baixa eficiência operacional [18]. Essa baixa eficiência pode ser representada pelo tempo de execução das soldagens que, para o modelo de painel teste adotado utilizando o passe reverso, levou de duas a três horas a mais do que o painel teste com passe contínuo. Dessa forma, mesmo apresentando valores baixos de distorção, quase se igualando à variável mudança de direção, dificilmente seria utilizado em uma soldagem na indústria, na qual existe uma maior complexidade na geometria e confecção dos painéis navais.

\subsubsection{Distorção Relacionada com Mudança da Sequência de Soldagem}

Neste trabalho não ocorreu a busca da melhor sequência de soldagem, mas sim saber qual a melhor dentre as utilizadas. Foi visualizado nos testes que a mudança da sequência surtiu pouco efeito sobre a distorção. Este fato vai de acordo com os estudos de [21], que comprovaram que, independente da sequência adotada, sempre existirão distorções. Das sequências utilizadas na segunda etapa, a que teve melhores resultados foi a sequência 2, que procurou restringir primeiramente cada quadrante, partindo da linha neutra do painel teste antes de executar a soldagem do lado posterior dos enrijecedores.

$\mathrm{Na}$ Figura 15, cada par de experimentos corresponde à soldagem de um parâmetro adotado nas etapas 1 e 2 , conforme o fluxograma da Figura 4 . Tomando como base os resultados apresentados na Figura 15, percebe-se que o quadrante três foi o que proporcionou os maiores valores de distorção em quase todos os experimentos, exceto para os experimentos 3 e 4, realizados com a mudança de direção de soldagem, e os experimentos 9 e 10 realizados com a utilização do passe reverso. Os experimentos 3 e 4 foram os que resultaram em uma melhor planicidade do painel teste. Realizados os experimentos da segunda etapa, ocorreu a tomada dos valores de distorção global dos painéis, dessa forma, foi idealizada uma proposta para a soldagem da terceira etapa.

\subsubsection{RESULTADOS DA TERCEIRA ETAPA}

Um dos objetivos desta etapa foi a formulação de uma proposta para a soldagem do painel otimizado. Outro objetivo foi a formulação do pacote operacional para os processos MAGSTT e MAG-CW vistos nos itens 2.1.2 e 2.1.3. Sabendo que no estudo realizado a sequência não exerceu forte influência 

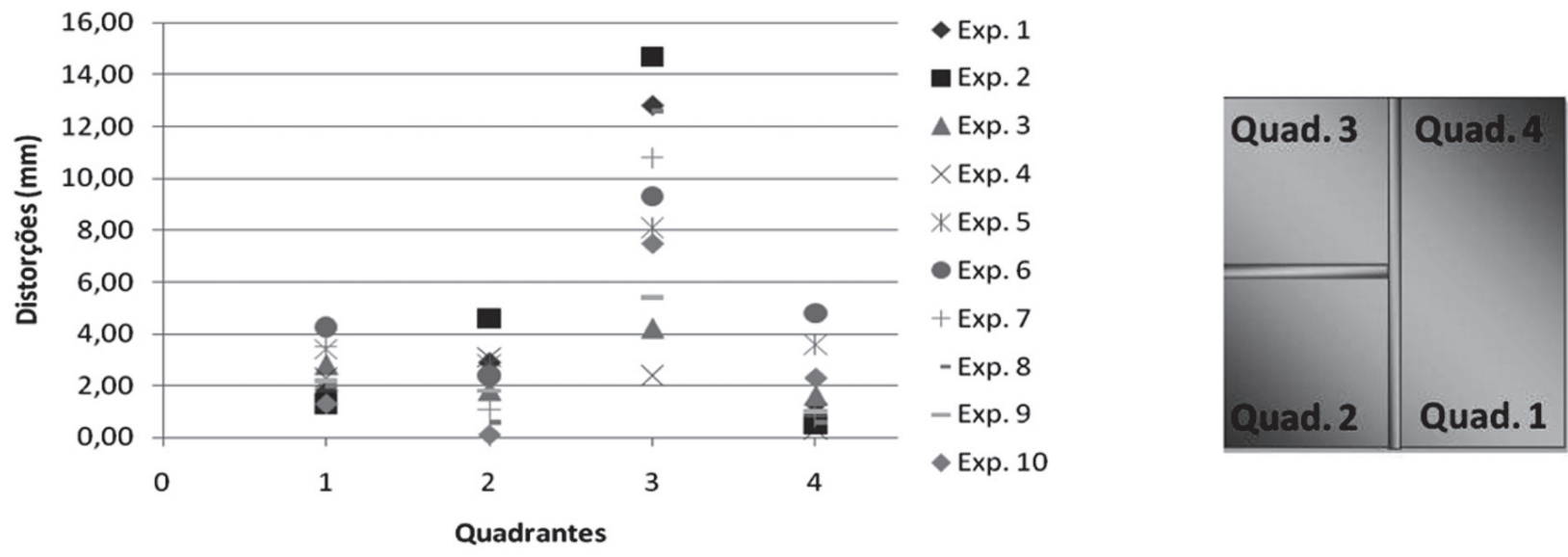

Figura 15. Distorção dos experimentos da segunda etapa por quadrante

nos resultados, foi proposta a utilização de uma sequência que possuísse fácil execução com relação ao arranjo da célula de trabalho e promovesse mais liberdade para o sistema soldado, e uma direção de soldagem que gerasse uma melhor planicidade dos experimentos da segunda etapa.

A sequência dois (Figura 8) foi selecionada por preencher as solicitações desejadas e a direção do centro do painel para as extremidades foi selecionada devido ter sido a variável que mais influenciou nos experimentos. Com base nessas exigências, a variável operacional apresentou-se da seguinte forma: Sequência dois $>$ Passe para fora do painel $>$ Passe contínuo.

A Tabela 5 apresenta as variáveis de análise da terceira etapa.

\begin{tabular}{|c|l|c|}
\hline Exp. & Variáveis & Processo \\
\hline 5 & $\begin{array}{l}\text { Sequência Dois/ Para Fora do Painel/ } \\
\text { Passe Contínuo }\end{array}$ & MAG-CC \\
\hline 7 & $\begin{array}{l}\text { Sequência Dois/ Para Fora do Painel// } \\
\text { Passe Contínuo }\end{array}$ & MAG-STT \\
\hline 8 & $\begin{array}{l}\text { Sequência Dois/ Para Fora do Painel/ } \\
\text { Passe Contínuo }\end{array}$ & MAG-CW \\
\hline
\end{tabular}

O processo de soldagem MAG-STT, em geral, teve pouca incidência de salpicos, característica atribuída ao controle interno da corrente, que proporciona uma transferência mais suave e constante, [7]. O processo de soldagem MAG-CW teve incidência de salpicos, contudo, uma quantidade menor que a obtida pelo processo MAG-CC, possivelmente devido a uma interação na região do arco voltaico durante a fusão dos dois arames. Analisando os valores de tensão reais obtidos através do sistema de aquisição de dados, observou-se que se manteve o mesmo comprimento de arco, ou seja, a tensão não variou, mesmo com a injeção de mais um arame na região do arco voltaico, não ocorreu alteração no comprimento do arco, mantendo-se em $21 \mathrm{~V}$.

Foi observado durante o processo de soldagem que, ao se injetar o arame não energizado na poça de fusão, era exercida uma força contrária ao movimento, agindo diretamente sobre o sistema tocha/braço robótico. Esse fato já tinha sido observado nos estudos de [17], cujo o processo de soldagem foi aplicado na forma semi-automática. Como o processo foi executado através de um braço robótico, essa força contrária não foi suficiente para alterar a DBCP e, consequentemente, o comprimento de arco.

Analisando os resultados da distorção global dos painéis teste da terceira etapa, Figura 16, observa-se que a distorção para o processo MAG-CC é semelhante ao processo MAG-STT, indo contra a informação dada pela literatura, [7], que atribui valores mais baixos de distorção, comparando com o processo MAG$\mathrm{CC}$, devido ao controle dos níveis de corrente, gerando menores valores de energia durante a soldagem. O processo MAG-CW obteve um nível de distorção aproximadamente $20 \%$ menor que os valores obtidos pelos processos MAG-CC e MAG-STT.

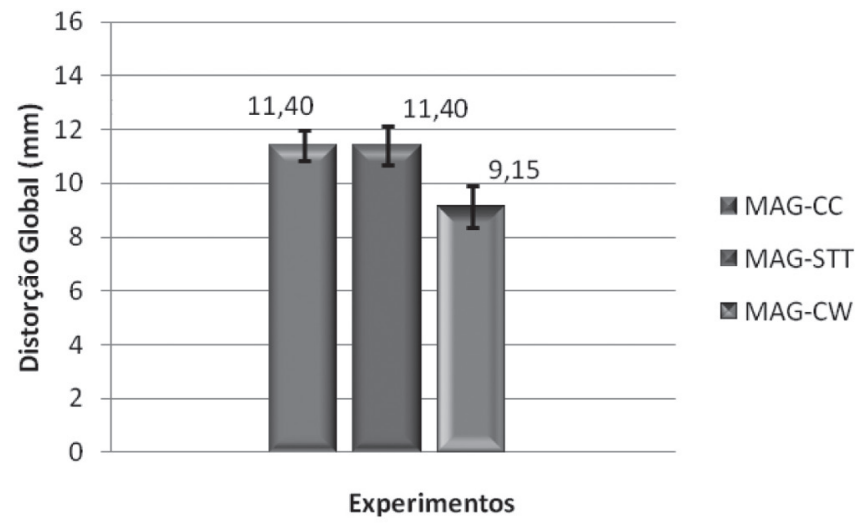

Figura 16. Resultados de distorção da terceira etapa dos experimentos

Percebeu-se que o processo MAG-CW promoveu valores de distorções mais homogêneos, ou seja, os valores de distorção resultaram em um painel mais plano que os painéis soldados com os outros dois processos.

$\mathrm{O}$ processo que obteve maiores valores de distorção foi o processo MAG-CC. Logo em seguida vem o processo MAGSTT, que apresentou uma tendência semelhante. 


\subsection{Considerações sobre o processo de MAG-CW}

Os resultados das soldagens com o processo MAG-CW foram muito bons, proporcionando uma regularidade de transferência metálica, comprovada "visualmente" e através dos oscilogramas dos valores de corrente adquirida. Foram retiradas quatro amostras das peças teste para cada processo utilizado com o intuito de se realizar a análise da área depositada dos cordões. Dessa forma, constatou-se que a área do processo MAG-CW era aproximadamente $22 \%$ menor do que a área obtida pelos processos MAG-CC e MAG-STT. Analisando a Figura 17, percebeu-se que os cordões depositados pelo processo MAG$\mathrm{CW}$ tiveram o menor valor de desvio padrão para a área obtida, possivelmente devido ao aspecto mais homogêneo do cordão depositado.

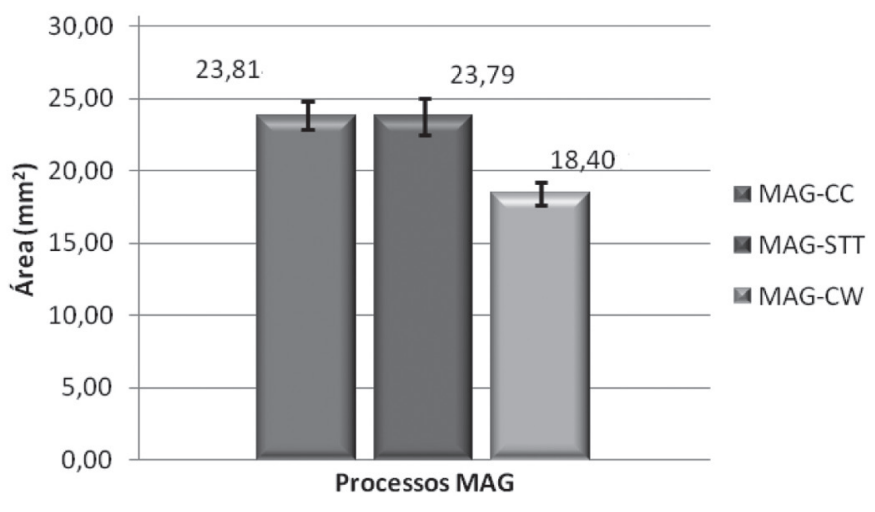

Figura 17. Áreas da seção transversal dos cordões dos painéis teste

Em síntese, o processo MAG-CW apresentou o "menor" resultado de distorção dentre os processos analisados neste estudo, não satisfazendo um dos critérios propostos para a realização da comparação dos valores de distorção, que seria manter o mesmo volume depositado de material. Os cálculos para encontrar a Relação de Deposição seguiram o mesmo procedimento para os processos MAG-CC e MAG-STT, para isso, foi utilizada a somatória das velocidades de alimentação (arame energizado + arame não energizado).

Para os processos MAG-STT e MAG-CC a relação teve êxito, todavia, essa relação não rege o princípio de funcionamento do sistema MAG-CW. Muito provavelmente houve a existência de fatores que agiram para que essa soma das velocidades não fosse usual. Uma possível explicação para esse menor valor de área depositada seria devido à diferença de velocidade do arame não energizado na entrada e na saída do cabeçote de alimentação, nesse caso a força que está sendo exercida sobre o arame não energizado está ocasionando essa diferença. Outro ponto que pode ser discutido é o aporte líquido de calor (energia imposta) para cada processo utilizado neste estudo, apresentado na Figura 18.

Como pode ser observado, o aporte de calor líquido do processo MAG-STT, Figura 18, é inferior ao obtido pelo processo MAG-CC, contudo, os valores médios de distorção global apresentaram-se praticamente iguais. Esse fato comprova que, quando empregados procedimentos adequados para uma soldagem (sequência e direção de soldagem, forma de fixação, etc.), é possível reduzir os valores de distorção provenientes do processo de soldagem com maior energia líquida. Vale a pena ressaltar que o aporte de calor não depende somente de parâmetros elétricos, mas também da técnica de soldagem, [22].

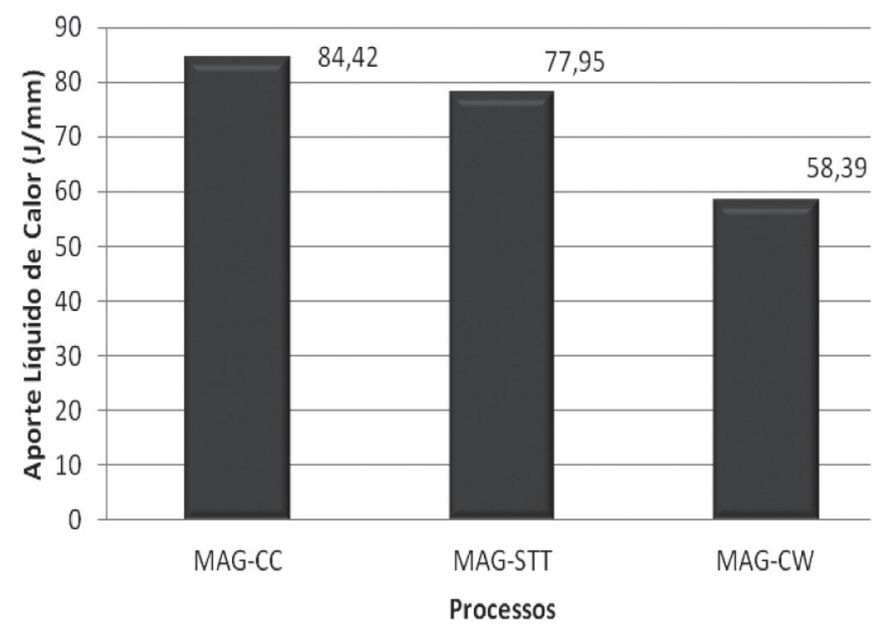

Figura 18. Resultados de aporte líquido de calor para os processos analisados

Ainda observando a Figura 18, pode-se supor que de posse de baixos níveis de energia líquida, são obtidas estruturas cristalinas menos afetadas metalurgicamente [23], o que se pressupõe que o processo MAG-CW resultará em uma melhor estrutura metalúrgica em relação ao processo MAG-CC e MAG-STT. Com relação ao menor volume de material depositado pelo processo MAG-CW, não se pode descartar totalmente os resultados de distorção obtidos, Figura 16, pois com a injeção de mais um arame na região do arco voltaico, a energia empregada no processo de soldagem irá diminuir, dessa forma, menores valores de energia líquida poderão gerar menores tensões residuais e, consequentemente, menores valores de distorção.

\section{Conclusões}

Foi observado nesse estudo que, quando empregados procedimentos adequados e parâmetros de soldagem otimizados, pode-se obter baixos níveis de distorção ao longo da estrutura soldada. As sequências de soldagem selecionadas não tiveram influência significativa sobre os valores de distorção global.

A mudança de direção foi a variável que exerceu a maior influência sobre os valores de distorção mensurados neste estudo. A utilização do passe reverso também exerceu influência sobre os valores de distorção, chegando a bons resultados. Todavia, não se mostrou aplicável devido ao grande tempo requerido para a execução da união dos elementos.

O processo MAG-CC apresentou os maiores valores de distorção relacionada neste estudo, tanto na análise global como na análise por quadrante do painel teste.

A utilização do processo MAG-STT apresentou resultados de distorções semelhantes aos obtidos pelo processo MAG-CC, ou seja, não mostrou influência sobre os valores de distorção global. 
$\mathrm{O}$ processo MAG-CW apresentou os menores valores de distorção neste estudo, tanto na análise global como na análise por quadrante, contudo, não manteve o mesmo volume depositado de material como era esperado ao utilizar-se a relação de deposição.

\section{Agradecimentos}

À Universidade Federal de Uberlândia, através do Laboratório de Pesquisa e Desenvolvimento de Processos de Soldagem, Laprosolda, onde realizei meus experimentos. Gostaria de agradecer aos professores Américo Scotti e Valtair Ferraresi pelo auxílio, e ao meu orientador Eduardo Braga, da Universidade Federal do Pará, e à Capes e o CNPQ pelo aporte financeiro.

\section{Referências Bibliográficas}

[1] ESTEFEN, S. et al. Análise de Evolução do Estado das Tensões Residuais de Soldagem. In: Conferência de Tecnologia de Soldagem e Inspeção. Centro de Tecnologia SENAI- RJ CTS Solda, 2008.

[2] SOARES, H. G. Estudo de Sequências de Soldagem para Redução e Eliminação de Distorções. 2006. 95p. Dissertação (Mestrado em Engenharia Mecânica) - Universidade Federal de Minas Gerais, Belo Horizonte.

[3] CABRAL, T. S. et al. Influência dos Procedimentos e Processos de Soldagem MAG-CC e MAG-STT na Redução de Deformações em Painéis de Estruturas Navais. In: XXXVII Congresso Nacional de Soldagem, CONSOLDA 2011. Outubro, Natal, RN.

[4] MACHADO, I. G. Soldagem e Técnicas Conexas: processos. Porto Alegre: editado pelo autor, 1996. 477p.

[5]WEMAN, K.; LINDÉM, G. - MIG Welding Guide. $1^{\text {a }}$ Ed. Edited by Woodhead Publishing Limited, Abington Hall, Abington, Cambridge CB1 6AH, England. 2006. 303p.

[6] FILHO, D. F. - Influência do Gás de Proteção na Soldagem MIG/MAG do Aço Inoxidável Ferrítico com Arames Ferríticos. 2007. 110p. Dissertação (Mestrado em Engenharia Mecânica) Universidade Federal de Uberlândia, Uberlândia.

[7] DERUNTZ, B. D, Assessing the Benefits of Surface Tension Transfer Welding. in: Industry-journal of Industrial Technology. Volume 19, Number 4, October. 2003.

[8] PARDO, E.; WECKMAN, D. C. Prediction of Weld Pool and Reinforcement Dimensions of GMA Welds Using a FiniteElement Model. Metallurgical Transactions. Volume 20B, p. 937-947, Dec. 1989.

[9] ASM HANDBOOK. Welding, Brazing and Soldering. ASM, 1993. Volume 6.

[10] MIRANDA, H. C. Influência da Indutância na Soldagem MIG/MAG. 1999. 204p. Dissertação (Mestrado em Engenharia Mecânica) - Universidade Federal de Uberlândia, Uberlândia.

[11] KOU, S. Welding Metallurgy. $2^{\text {a }}$ Ed. Edited, United States of America: Wiley-Interscience publication, 2003. 466p.

[12] WU, C. S. et al. Numerical Analysis of Double-Electrode Gas Metal Arc Welding Process. Computational Materials Science, Volume 39, p. 416-423, Mar. 2006.
[13] SOUZA, D. Levantamento de Mapas Operacionais de Transferência Metálica para Soldagem MIG/MAG de Aço ao Carbono na Posição Plana. 2010. 286 p. Dissertação (Mestrado em Engenharia Mecânica) - Universidade Federal de Uberlândia, Uberlândia.

[14] REZENDE, G. M. et al. Avaliação de Uma Metodologia para Seleção de Parâmetros em Soldagem MIG/MAG por Curto-Circuito. In: VI CONGRESSO NACIONAL DE ENGENHARIA MECÂNICA, CONEM. ABCM. Agosto, Campina Grande, Paraíba.

[15]LINCOLN ELECTRIC - Surface Tension Transfer (STT), STT Open Root. Disponível em $<$ http://content.lincolnelectric. com/pdfs/products/literature/nx310.pdf $>$. Acesso em: 7 Maio 2010.

[16] SÁBIO, A. D. Estudo da Viabilidade Operacional do Processo de Soldagem MAG com Alimentação Adicional de Arame Frio. 2007. 147p. Dissertação (Mestrado em Engenharia Mêcanica) - Universidade Federal do Pará, Belém.

[17] SILVA, F. G. Estudo da Viabilidade do Processo FCAW com adição de arame Frio Aplicado a Indústria Naval. 2010. 152p. Dissertação (Mestrado em Engenharia Mecânica) Universidade Federal do Pará, Belém.

[18]OKUMURA, T.; TANIGUCHI, C. Engenharia de Soldagem e Aplicações. $1^{\text {a }}$ Ed. Rio de Janeiro: LTC Editora, 1982. 461p.

[19] TSAI, C. L.; PARK, S. C.; CHENG. W. T. Welding Distortion of a Thin-Plate Panel Structure. The effect of welding sequence on panel distortion is evaluated. Welding Research Supplement, p. 156-165, May 1999.

[20]FENG, Z. Processes and Mechanisms of Welding Residual Stress and Distortion. $1^{\text {a }}$ Ed. Cambridge England, Woodhead Publishing and Maney Publishing on behalf of The Institute of Materials, Minerals \& Mining. 2005. 353p.

[21] SATTARI, I.; JAVADI, Y. Influence of Welding Sequence on Welding Distortions in Pipes. International Journal of Pressure Vessels and Piping. Volume 85, p. 265-274, 2008.

[22]ZEEMANN, A. Energia de Soldagem. Rio de Janeiro (2003). Disponível em: <http://www.infosolda.com.br/ artigos/ metsol05.pdf>. Acesso em: 02 Maio 2009.

[23] MAGALHÃES, R. Avaliação do uso de Extensometria na Análise de Tensões Residuais na Soldagem. 2008. 129 p. Dissertação (Mestrado em Mecatrônica) - Universidade Federal da Bahia, Salvador. 